\title{
Explaining the Supernova Data without Accelerating Expansion
}

\author{
W.M. Stuckey ${ }^{1}$, T.J. McDevitt ${ }^{2}$ and M. Silberstein ${ }^{3}$ \\ 1 Department of Physics \\ Elizabethtown College \\ Elizabethtown, PA 17022 \\ stuckeym@etown.edu \\ 2 Department of Mathematical Sciences \\ Elizabethtown College \\ Elizabethtown, PA 17022 \\ mcdevittt@etown.edu \\ ${ }^{3}$ Department of Philosophy and Foundations of Physics \\ Committee for Philosophy and the Sciences \\ University of Maryland \\ College Park, MD 20742 \\ msilbers@umd.edu
}

\begin{abstract}
The 2011 Nobel Prize in Physics was awarded "for the discovery of the accelerating expansion of the Universe through observations of distant supernovae." However, it is not the case that the type Ia supernova data necessitates accelerating expansion. Since we do not have a successful theory of quantum gravity, we should not assume general relativity (GR) will survive unification intact, especially on cosmological scales where tests are scarce. We provide a simple example of how GR cosmology may be modified to produce a decelerating Einstein-de Sitter cosmology model (EdS) that accounts for the Union2 Compilation data as well as the accelerating $\Lambda \mathrm{CDM}$ (EdS plus a cosmological constant).
\end{abstract}

Essay written for the Gravity Research Foundation 2012 Awards for Essays on Gravitation

Submitted 15 March 2012 


\section{Introduction}

It is widely acknowledged that the universe is undergoing accelerating expansion as evidenced by type Ia supernova data[1]. Indeed, the 2011 Nobel Prize in Physics was awarded specifically "for the discovery of the accelerating expansion of the Universe through observations of distant supernovae" [2]. Of course, cosmological data isn't literally "expansion velocity versus time" whence one reads off acceleration or deceleration. The Union2 Compilation of type Ia supernova data, for example, provides distance modulus $\mu$ and redshift $z$ for each supernova. In order to find $\mu$ versus $z$ for a given cosmology model, we first find proper distance as a function of $z$, then compute the luminosity distance $D_{L}$, and finally

$$
\mu=5 \log \left(\frac{D_{L}}{10 \mathrm{pc}}\right)
$$

Each cosmology model will, in general, have different forms for proper distance as a function of $z$ and $D_{L}$ as a function of proper distance. Thus, the kinematical conclusion of accelerating expansion comes indirectly from the cosmology model that produces the best fit to $\mu$ versus $z$ (as long as it's in compliance with other cosmological data as well, of course). That model is currently $\Lambda$ CDM, which simply adds a cosmological constant $\Lambda$ to the Einstein-de Sitter cosmology (EdS) model $\left(\Omega_{M}+\Omega_{\Lambda}=1\right)[3][4][5]$. Since $\Lambda$ produces accelerating expansion, the conclusion in the Nobel citation follows.

While $\Lambda$ CDM does work well in the context of all cosmological data, it introduces a new "problem," i.e., we have no explanation for a cosmological constant of the size needed in $\Lambda$ CDM [6][7][8]. This, in part, has spawned alternatives to $\Lambda$ CDM. Yousaf Butt at the Harvard-Smithsonian Center for Astrophysics writes[9]

Various alternatives to an accelerating universe have also been proposed (see, for example, C. Tsagas, Phys. Rev. D 84, 063503 (2011)). Whether such alternatives are viable remains to be seen, but the Nobel Committee for Physics has perhaps acted somewhat prematurely by selecting a preferred interpretation of the supernova projects' data. The effect, intentional or not, is to bully the skeptics into silence, self-censorship, or ridicule, whereas good science proceeds with a healthy dose of skepticism and with open minds.

Even Perlmutter, a co-recipient of the 2011 Nobel Prize in Physics, said[10]

Not only do we not know what dark energy might be, that would be making the universe expand faster and faster, we don't even know whether really the answer will turn out to be a new energy in the universe. It's possible that we've just discovered an extra wrinkle in Einstein's Theory of Relativity, and that that would be the real final result.

While the most popular attempt at explaining the supernova data without accelerating expansion (inhomogeneous spacetimes[11][12][13][14]) and, therefore, without $\Lambda$ has not been ruled out, the possibility is increasingly constrained by WMAP and Hubble data[15]. Herein, we introduce an alternative to $\Lambda$ CDM that is compatible with a current 
(2011) "best estimate" for the Hubble constant[16], as well as the facts that space is flat and sources are distributed (roughly) isotropically per WMAP[17]. In this model, the universe is decelerating and contains no "new energy."

\section{The Model}

After decades of effort by some of the greatest minds in physics, we still have no generally accepted theory of quantum gravity or unified physics. Of course, there are any number of reasons this might be the case, but consider the possibility that we've been trying to quantize the wrong classical theory of gravity. That is, perhaps general relativity (GR) is only an approximation to the correct classical theory of gravity, as evidenced by the supernova data. We were motivated to consider this possibility by our work on foundational issues[18][19][20], so this was not an ad hoc response to the supernova data. Since our foundational method employs a graphical path integral approach to quantum physics, we are motivated to pursue and modify a graphical, least action approach to GR, i.e., Regge calculus[21][22][23][24].

Specifically, we constructed a Regge differential equation for the time evolution of the scale factor $a(t)$ in EdS and made two modifications (for details see our recent paper[25] in Classical and Quantum Gravity). First, we allowed spatial links of the Regge graph to be large (as defined below) in accord with the assumption that Regge calculus is fundamental while GR is the continuous approximation thereto. Second, we did not assume that luminosity distance $D_{L}$ is trivially related to graphical proper distance $D_{p}$ between photon receiver and emitter as it is in EdS, i.e., in $\operatorname{EdS} D_{L}=(1+z) d_{p}$ where $d_{p}$ is proper distance between photon receiver and emitter. There are two reasons we did not make this assumption. First, in our foundational view, space, time and sources are co-constructed when rendering the transition amplitude, yet $D_{p}$ is found without taking into account EM sources responsible for $D_{L}$. That is to say, in Regge EdS (as in EdS) one assumes that pressureless dust dominates the stress-energy tensor and is exclusively responsible for the graphical notion of spatial distance $D_{p}$. However, even though the EM contribution to the stress-energy tensor is negligible, EM sources are being used to measure the spatial distance $D_{L}$, so in our foundational view the EM interaction should contribute to the spacetime metric. Second, in our view, there are no "photon paths being stretched by expanding space," so we cannot simply assume $D_{L}=(1+z) D_{p}$ as in EdS. Indeed, we find the trivial EdS relationship between luminosity distance and proper distance holds only when $D_{p}$ is small on cosmological scales. In order to generate a relationship between $D_{L}$ and $D_{p}$, we turned to the self-consistency equation $K Q=J$ in our foundational approach to physics[19], where $K$ is the differential operator, $Q$ is the 'field' and $J$ is the source. Since we want a relationship between $D_{L}$ and $D_{p}$, the 'field' of interest is a metric $h_{\alpha \beta}$ relating the graphical distance $D_{p}$, obtained theoretically using no EM sources, to the luminosity distance $D_{L}$, obtained observationally via EM sources. The region in question (inter-nodal region between emitter and receiver) has metric $\eta_{\alpha \beta}$ given by $d s^{2}=-c^{2} d t^{2}+d D_{p}^{2}$, so the inner product 
of interest can be written $\eta_{\alpha \beta}+h_{\alpha \beta}$ where the spatial coordinate is $D_{p}$. Since each EM source proper is not "stretched out" by the expansion of space, the spatiotemporal relationship between emitter and receiver is modeled per this inter-nodal region alone. Thus, unlike EdS, we have no a priori basis to relate $D_{L}$ to $D_{p}$, so we begin with the assumption $D_{L}=(1+z) \sqrt{\overrightarrow{D_{p}} \cdot \overrightarrow{D_{p}}}=(1+z) D_{p} \sqrt{1+h_{11}}$, where $\overrightarrow{D_{p}}=\left(0, D_{p}\right)$.

The specific form of $K Q=J$ that we used was borrowed from linearized gravity in the harmonic gauge, i.e., $\partial^{2} h_{\alpha \beta}=-16 \pi G\left(T_{\alpha \beta}-\frac{1}{2} \eta_{\alpha \beta} T\right)$. We emphasize that $h_{\alpha \beta}$ here corrects the graphical inner product $\eta_{\alpha \beta}$ in the inter-nodal region between the worldlines of photon emitter and receiver, where $\eta_{\alpha \beta}$ is obtained via a matter-only stress-energy tensor. Since the EM sources are negligible in the matter-dominated solution, we have $\partial^{2} h_{\alpha \beta}=0$ to be solved for $h_{11}$. Obviously, $h_{11}=0$ is the solution that gives the trivial relationship used in EdS, but allowing $h_{11}$ to be a function of $D_{p}$ allows for the possibility that $D_{L}$ and $D_{p}$ are not trivially related. We have $h_{11}=A D_{p}+B$ where $A$ and $B$ are constants and, if the inner product is to reduce to $\eta_{\alpha \beta}$ for small $D_{p}$, we have $B=0$. Presumably, $A$ should follow from the corresponding theory of quantum gravity, so an experimental determination of its value provides a guide to quantum gravity per our view of classical gravity. Our modified Regge calculus (MORC) best fit to the Union2 Compilation data gives $A^{-1}=8.38 \mathrm{Gcy}$, so the correction to $\eta_{11}$ is negligible except at cosmological distances, as expected. Essentially, we're saying the supernova data is an indication that $A \neq 0$ so that one cannot simply assume the distance $D_{L}$ measured using EM sources corresponds trivially to the graphical distance $D_{p}$ even though the EM sources contribute negligibly to the stress-energy tensor.

Our Regge EdS equation for the time evolution of the scale factor $a(t)$ is

$$
\frac{\pi-\cos ^{-1}\left(\frac{v^{2} / c^{2}}{2\left(v^{2} / c^{2}+2\right)}\right)-2 \cos ^{-1}\left(\frac{\sqrt{3 v^{2} / c^{2}+4}}{2 \sqrt{v^{2} / c^{2}+2}}\right)}{\sqrt{v^{2} / c^{2}+4}}=\frac{G m}{2 r c^{2}}
$$

where $r=R a(t), R$ is the internodal distance today, and $v=d r / d t$. If $v^{2} / c^{2} \ll 1$, then a power series expansion of the LHS of Equation (2) gives

$$
\frac{v^{2}}{4 c^{2}}+\mathcal{O}\left(\frac{v}{c}\right)^{4}=\frac{G m}{2 r c^{2}}
$$

Thus, to leading order, our Regge EdS is EdS, i.e., $\frac{v^{2}}{2}=\frac{G m}{r}$, which is just a Newtonian conservation of energy expression for an unit mass moving at escape velocity $v$ at distance $r$ from mass $m$. In Regge EdS, $v$ is the relative 'Newtonian' velocity of spatially adjacent nodes of mass $m$. So, spatial links are "small" when the 'Newtonian' graphical velocity $v$ between spatially adjacent nodes on the Regge graph is small compared to c. In that case the dynamics between adjacent spatial nodes is just Newtonian and the evolution of $a(t)$ in Regge EdS is equal to that in EdS. For the best fit MORC trial, the use of large spatial links did make a small difference (Figure 1), but generally the Regge evolution played a minor role in the MORC fits, so the major factor in improving EdS was $D_{L}=(1+z) D_{p} \rightarrow D_{L}=(1+z) \sqrt{\overrightarrow{D_{p}} \cdot \overrightarrow{D_{p}}}$. 


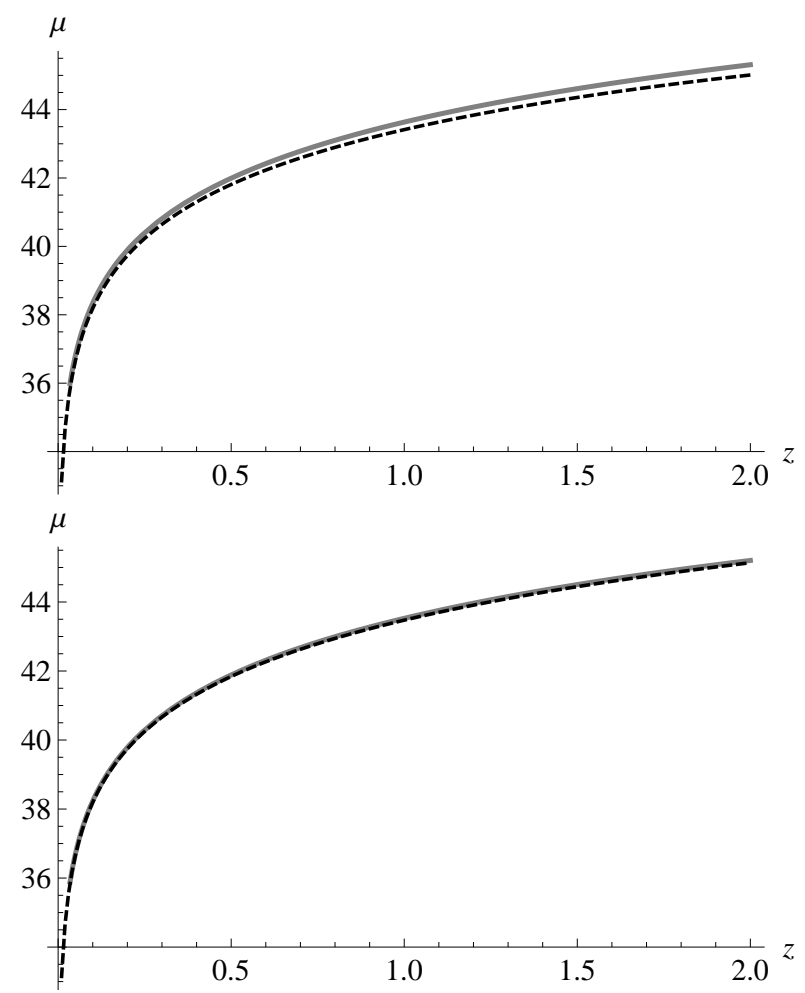

Figure 1. Top graph shows Regge evolution (dotted) without the $h_{11}$ correction and EdS evolution (gray) for the best fit MORC trial, i.e., $\mathrm{SSE}=1.77$. The bottom graph makes the same comparison for a trail in which $\mathrm{SSE}=1.78$.

\section{The Fit}

We fit EdS, MORC, and $\Lambda$ CDM to the Union2 Compilation data[26] (Figures 2 and 3). In order to establish a statistical reference, we first found that a best fit line through $\log \left(\frac{D_{L}}{\mathrm{Gpc}}\right)$ versus $\log z$ gives a correlation of 0.9955 and a sum of squares error (SSE) of 1.95. EdS cannot produce a better fit than this best fit line. The best fit EdS gave $\mathrm{SSE}=2.68$ using $H_{o}=60.9 \mathrm{~km} / \mathrm{s} / \mathrm{Mpc}$. Both MORC and $\Lambda$ CDM produced better fits than the best fit line. The best fit $\Lambda$ CDM gave SSE $=1.79$ using $H_{o}=69.2 \mathrm{~km} / \mathrm{s} / \mathrm{Mpc}$, $\Omega_{M}=0.29$ and $\Omega_{\Lambda}=0.71$. This best fit $\Lambda \mathrm{CDM}$ is consistent with its fit to the WMAP data[17]. The best fit MORC gave $\mathrm{SSE}=1.77$ and $H_{o}=73.9 \mathrm{~km} / \mathrm{s} / \mathrm{Mpc}$ using $R=A^{-1}$ $=8.38 \mathrm{Gcy}$ and a nodal mass $m=1.71 \times 10^{52} \mathrm{~kg}$. Both MORC and $\Lambda$ CDM are within two sigma of a current (2011) "best estimate" for the Hubble constant of $H_{o}=(73.8 \pm$ 2.4) $\mathrm{km} / \mathrm{s} / \mathrm{Mpc}$ [16], while EdS lies over five sigma out. Given the scatter in the data, MORC and $\Lambda$ CDM produce essentially equivalent fits, clearly superior to EdS. 


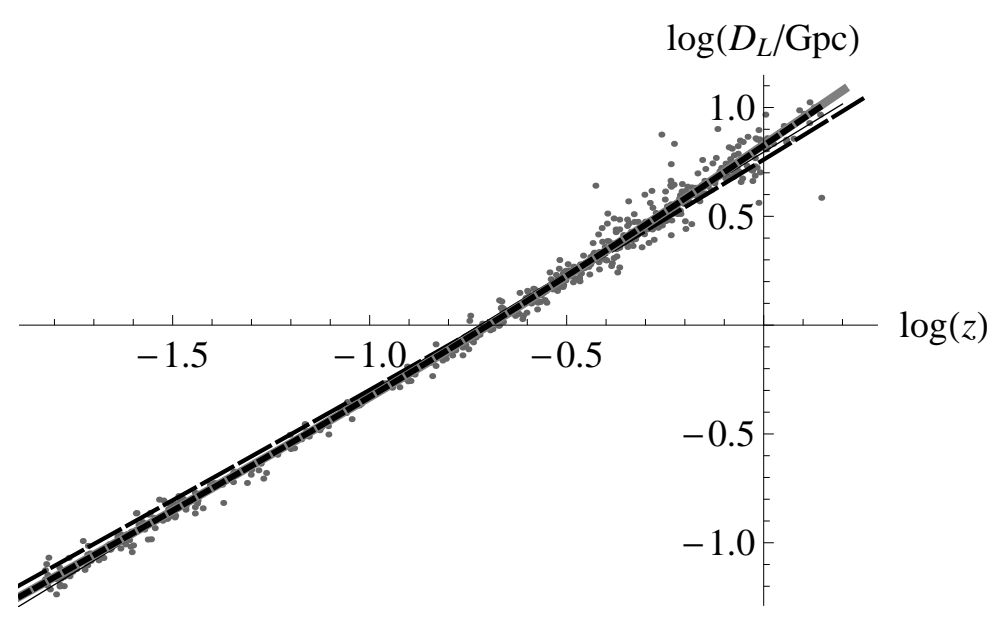

Figure 2. Plot of transformed Union2 data along with the best fits for linear regression (thin black), EdS (dashed), $\Lambda \mathrm{CDM}$ (gray), and MORC (dotted).

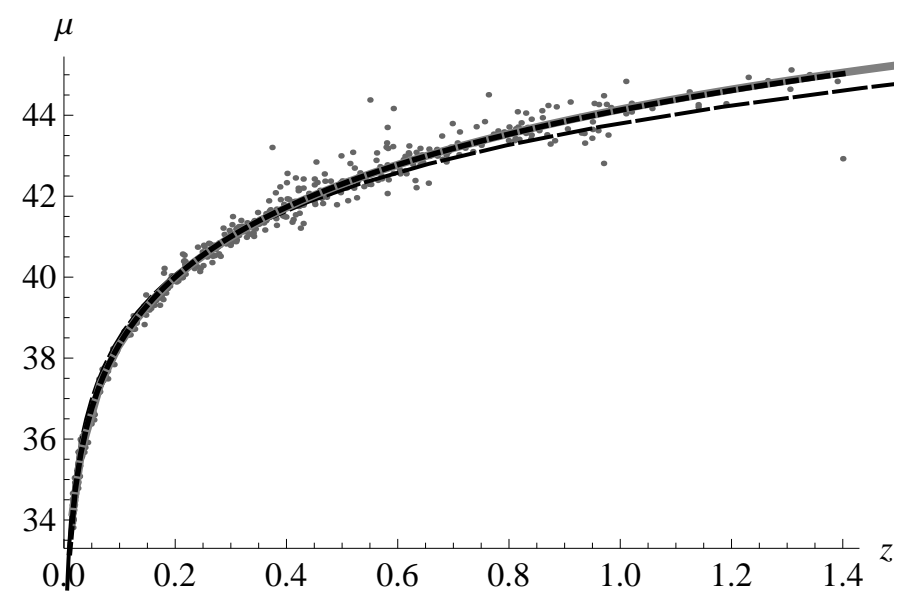

Figure 3. Plot of Union2 data along with the best fits for EdS (dashed), $\Lambda$ CDM (gray), and MORC (dotted). The MORC curve is terminated at $z=1.4$ in this figure so that the $\Lambda$ CDM curve is visible underneath.

\section{Conclusion}

We have shown that a modified Regge calculus (MORC) version of Einstein-de Sitter cosmology (EdS) produces a fit of the Union2 Compilation of type Ia supernova data equaling that of $\Lambda$ CDM. MORC's value of the Hubble constant is within one sigma of a current (2011) "best estimate" and its distribution of sources in flat space is consistent with WMAP data. Thus, the supernova data may point to such "an extra wrinkle in Einstein's Theory of Relativity" and, since MORC models a decelerating universe (without "new energy"), it may well be premature to claim "the discovery of the accelerating expansion of the Universe through observations of distant supernovae." 


\section{References}

[1] Perlmutter, S.: Supernovae, Dark Energy, and the Accelerating Universe. Physics Today, 53-60 (April 2003)

[2] http://www.nobelprize.org/

[3] Riess, A.G., Filippenko, A.V., Challis, P., Clocchiattia, A., Diercks, A., Garnavich, P.M., Gilliland, R.L., Hogan, C.J., Jha, S., Kirshner, R.P., Leibundgut, B., Phillips, M.M., Reiss, D., Schmidt, B.P., Schommer, R.A., Smith, R.C., Spyromilio, J., Stubbs, C., Suntzeff, N.B., Tonry, J.: Observational Evidence from Supernovae for an Accelerating Universe and a Cosmological Constant. Astronomical Journal 116, 1009-1038 (1998). astro-ph/9805201

[4] Perlmutter, S., Aldering, G., Goldhaber, G., Knop, R.A., Nugent, P., Castro, P. G., Deustua, S., Fabbro, S., Goobar, A., Groom, D. E., Hook, I. M., Kim, A. G., Kim, M. Y., Lee, J. C., Nunes, N. J., Pain, R., Pennypacker, C. R., Quimby, R., Lidman, C., Ellis, R. S., Irwin, M., McMahon, R. G., Ruiz-Lapuente, P., Walton, N., Schaefer, B., Boyle, B. J., Filippenko, A. V., Matheson, T., Fruchter, A. S., Panagia, N., Newberg, H. J. M., Couch, W. J.: Measurements of $\Omega$ and $\Lambda$ from 42 High-Redshift Supernovae. The Astrophysical Journal 517(2) 565-586 (1999)

[5] Suzuki, N., Rubin, D., Lidman, C., Aldering, G., Amanullah, R., Barbary, K., Barrientos, L., Botyanszki, J., Brodwin, M., Connolly, N., Dawson, K., Dey, A., Doi, M., Donahue, M., Deustua, S., Eisenhardt, P., Ellingson, E., Faccioli, L., Fadeyev, V., Fakhouri, H., Fruchter, A., Gilbank, D., Gladders, M., Goldhaber, G., Gonzalez, A., Goobar, A., Gude, A., Hattori, T., Hoekstra, H., Hsiao, E., Huang, X., Ihara, Y., Jee, M., Johnston, D., Kashikawa, N., Koester, B., Konishi, K., Kowalski, M., Linder, E., Lubin, L., Melbourne, J., Meyers, J., Morokuma, T., Munshi, F., Mullis, C., Oda, T., Panagia, N., Perlmutter, S., Postman, M., Pritchard, T., Rhodes, J., Ripoche, P., Rosati, P., Schlegel, D., Spadafora, A. Stanford, S., Stanishev, V., Stern, D., Strovink, M.,Takanashi, N., Tokita, K., Wagner, M., Wang, L., Yasuda, N., Yee, H.: The Hubble Space Telescope Cluster Supernova Survey: V. Improving the Dark Energy Constraints Above $z>1$ and Building an Early-Type-Hosted Supernova Sample. arXiv:1105.3470

[6] Carroll, S.: The Cosmological Constant. arXiv:astro-ph/0004075v2 (section 4)

[7] Weinberg, S.: The Cosmological Constant Problems. arXiv:astro-ph/0005265

[8] Bianchi, E., Rovelli, C., Kolb, R.: Is dark energy really a mystery? Nature 466, 321-322 (July 2010).

[9] Butt, Y.M.: Premature Nobel Prize decision? Physics Today 65(2), 10 (2012).

[10] Nobels Honor Discoveries of Accelerating Universe, Quasicrystals. APS News 20(10), 1 and 5 (2011).

[11] Garfinkle, D.: Inhomogeneous spacetimes as a dark energy model. Classical and Quantum Gravity 23, 4811-4818 (2006). arXiv:gr-qc/0605088v2

[12] Paranjape, A., Singh, T.P.: The Possibility of Cosmic Acceleration via Spatial Averaging in Lemaître-Tolman-Bondi Models. Classical and Quantum Gravity 23, 6955-6969(2006). arXiv:astro-ph/0605195v3

[13] Tanimoto, M., Nambu, Y.: Luminosity distance-redshift relation for the LTB solution near the center. Classical and Quantum Gravity 24, 3843-3857 (2007). arXiv:gr-qc/0703012

[14] Clarkson, C., Maartens, R.: Inhomogeneity and the foundations of concordance cosmology. Classical and Quantum Gravity 27, 124008 (2010). arXiv:1005.2165v2

[15] Zibin, J. P., Moss, A., Scott, D.: Can We Avoid Dark Energy? Physical Review Letters 101, $251303(2008)$

[16] Riess, A.G., Macri, L., Casertano, S., Lampeitl, H., Ferguson, H.C., Filippenko, A.V., Jha, S.W., Li, W., Chornock, R.: A 3\% Solution: Determination of the Hubble Constant with the Hubble Space Telescope and Wide Field Camera 3. The Astrophysical Journal 730, 119 (2011)

[17] Komatsu, E., Smith, K.M., Dunkley, J., Bennett, C.L., Gold, B., Hinshaw, G., Jarosik, N., Larson, D., Nolta, M.R., Page, L., Spergel, D.N., Halpern, N., Hill, R.S., Kogut, A., Limon, M., Meyer, S.S., Odegard, N., Tucker, G.S., Weiland, J.L., Wollack, E., and Wright, E.L.: Seven-Year 
Wilkinson Microwave Anisotropy Probe (WMAP) Observations: Cosmological Interpretation. arXiv:1001.4538v3

[18] Stuckey, W.M., Silberstein, M., Cifone, M.: Reconciling spacetime and the quantum: Relational Blockworld and the quantum liar paradox. Foundations of Physics 38(4), 348-383 (2008). quant$\mathrm{ph} / 0510090$

[19] Stuckey, W.M., Silberstein, M., McDevitt, T.: Relational Blockworld: A Path Integral Based Interpretation of Quantum Field Theory. quant-ph/0908.4348

[20] Silberstein, M., Stuckey, W.M., McDevitt, T.: Being, Becoming and the Undivided Universe: A Dialogue between Relational Blockworld and the Implicate Order Concerning the Unification of Relativity and Quantum Theory (2011). quant-ph/1108.2261. Under review at Foundations of Physics.

[21] Regge, T.: General relativity without coordinates. Nuovo Cimento 19, 558571 (1961)

[22] Misner, C.W., Thorne, K.S., Wheeler, J.A.: Gravitation. W.H. Freeman, San Francisco (1973), p 1166

[23] Barrett, J.W.: The geometry of classical Regge calculus. Classical and Quantum Gravity 4, $15651576(1987)$

[24] Williams, R.M., Tuckey, P.A.: Regge calculus: a brief review and bibliography. Classical and Quantum Gravity 9, 14091422 (1992)

[25] Stuckey, W.M., McDevitt, T., Silberstein, M.: Modified Regge calculus as an explanation of dark energy. Classical and Quantum Gravity 29, 055015 doi:10.1088/0264-9381/29/5/055015 (2012) gr-qc/1110.3973

[26] Amanullah, R., Lidman, C., Rubin, D., Aldering, G., Astier, P., Barbary, K., Burns, M.S., Conley, A., Dawson, K.S., Deustua, S.E., Doi, M., Fabbro, S., Faccioli, L., Fakhouri, H.K., Folatelli, G., Fruchter, A.S., Furusawa, H., Garavini, G., Goldhaber, G., Goobar, A., Groom, D.E., Hook, I., Howell, D.A., Kashikawa, N., Kim, A.G., Knop, R.A., Kowalski, M., Linder, E., Meyers, J., Morokuma, T., Nobili, S., Nordin, J., Nugent, P.E., Ostman, L., Pain, R., Panagia, N., Perlmutter, S., Raux, J., Ruiz-Lapuente, P., Spadafora, A.L., Strovink, M., Suzuki, N., Wang, L., Wood-Vasey, W.M., Yasuda, N., (The Supernova Cosmology Project): Spectra and Light Curves of Six Type Ia Supernovae at $0.511<z<1.12$ and the Union2 Compilation. The Astrophysical Journal 716, 712-738 (2010). astro-ph/1004.1711v1 